\title{
Benchmarking Commercial OCR Engines for Technical Drawings Indexing
}

\author{
J.C.Lecoq ${ }^{1}$, L.Najman ${ }^{2}$, O.Gibot ${ }^{2}$ and E.Trupin ${ }^{3}$ \\ 1 - PSI, Insa de Rouen - 76801 Saint-Etienne du Rouvray Cedex - France \\ jclecoq@servasi.insa-rouen.fr \\ 2 - Océ Industries - 1 rue Jean Lemoine - 94015 Créteil Cedex - France \\ laurent.najman@ocegr.fr - ogib@ ocegr.fr \\ 3 - PSI, Université de Rouen - F-76821 Mont-Saint-Aignan Cedex - France \\ Eric.Trupin@univ-rouen.fr
}

\begin{abstract}
The choice of a commercial Optical Character Recognition (OCR) engine is important for the process of automatically indexing technical drawings from their title blocks. We would like to benchmark commercial OCR engines with respect to their inclusion in the global digitalisation chain from scanning to understanding the text information contained in a technical drawing document. The crucial (costly) point is the manual correction of OCR recognition errors. By benchmarking, we intend to identify, for our application domain, the causes for OCR errors which are the most costly to correct.

For a given OCR engine, we model the correction cost as a function of image characteristics. Thus, our methodology relies on the two following issues:

On the one hand, the design of the correction cost, representing the difficulty of correction for a human operator.

On the other hand, the classification of image characteristics that may lead to OCR recognition errors. We choose to analyse the behaviour of this correction cost by Principal Component Analysis (PCA), comparing two by two the engines to discover their complementarity.
\end{abstract}

This methodology allows us to obtain a list of domaindependant problems for OCR engines, classified by importance with respect to the correction cost. This list could then be used to correctly choose the OCR engine, or to enhance the OCR execution, by focusing on the most important problems.

While we are confident it could easily be implemented for other document classes, we apply this methodology to the domain of technical drawings, and found that our OCR engines were not adapted to our problem.

\section{Introduction}

For automatically indexing a technical drawing from the content of the title block, we need first to identify the type of title block, then to extract the fields needed for indexing and to run the content of those fields to an OCR engine. This process is similar to indexing a form. While the identification and the extraction steps have been addressed elsewhere [6], we would like here to focus on the problem of the choice of the OCR engine for a correct recognition. 
A large number of (commercial) OCR engines are available for printed character recognition. Those OCR engines are powerful and include state-of-the-art technology for recognition. Thus, there is no need to develop a proprietary solution. However, when we come to the difficult question of choosing an OCR engine suited to a given task, we face a problem: OCR engines will always commit some errors, especially for our application domain on which they are not trained.

Commercial OCR engines are "black-boxes", i.e. we do not know how they take their decision on a given sample. To evaluate the performance of those OCRs, several benchmarking techniques have been designed [1, $2,7,8,9]$. In those benchmarks some engine's performances are expressed, generally as a recognition rate. However, from an industrial point of view, what is important is not the error itself, but the difficulty of correction of this error. For our application domain, it may be faster to manually enter the whole field if there are some recognition errors rather than trying to correct individually those errors.

To identify the reasons of error, we describe a sample of images of our domain by some parameters. Those parameters may prevent an OCR from recognising perfectly the images. The creation of a database of image description with such parameters will enable characterising strengths and weaknesses of OCR engines, through a statistical analysis.

Answers to those questions will enable to focus researches on identified topics. For instance, once we know the behaviour of some OCR engines on a given document domain, we can try to combine various OCR engines, by choosing those with different behaviours rather than assuming that the engines do not have the same behaviour. On the other hand, different combination strategies can be designed. For example, we can think of building some image characteristic estimators, which will allow choosing the correct OCR engine with respect to a given image.

This paper is organised as follows: the following two sections are devoted to the cost of correction, and to the image characteristics that may lead to OCR errors. In section 5, we describe the experiment and we analyse the results.

\section{$2 \quad$ Human Cost of Correction}

For a given OCR engine, we have to evaluate how much time it will take for a human operator to correct the result of an OCR interpretation of a given field. We first think of automating this process, using methods based on a correctly defined editing distance between the ground truth and the OCR result. It is not straightforward to design such a distance: we have to define the weight of a substitution, a deletion, or an insertion, then to apply a string-matching algorithm, which will allow computing the minimum number of edit operations required to correct the result. This minimum number could be used as a measure of the operator correction time.

But in our case, the difficulty is that the operator can choose to retype the whole field rather than trying to modify the OCR result. Thus, rather than trying to model the process, we choose to measure the level of difficulty for the operator to correct a field, and we fill up a database with this level. These correction costs permit to have a more realistic opinion concerning an expert evaluation. On the other hand, a string-matching algorithm does not reflect the real cost that an operator will have to correct results. Indeed, the values computed by such an algorithm only express a distance between two strings (ground trough and result), letter by letter, with a criteria of insertion/deletion/substitution. The human behaviour is not the same at all (learning, reasoning,...). For this reason, in the aim to get a computer cost that will express human efforts, we have chosen a four-class human cost of correction. The four classes are defined by rules, and can be summarised as: "Nothing" (the result is enough for the indexing); "A little" (low efforts, for example little words to be corrected); "Middle" (one or two numbers to correct in a identify number); "High" (sometimes better to entirely rewrite the words).

For the next parts, those cost classes will have the numbers 1 (low), 2 (little), 3 (middle), 4 (high).

\section{$3 \quad$ Image characteristics}

We start our Error Cause Classification from the works of [5], and integrate results from [4]. However, our application domain being different, our classification is different.

\subsection{Nagy, Nartker and Rice classification of error causes}

Nagy, Nartker and Rice [5] introduced a taxonomy of OCR error causes based on several factors. Their taxonomy addresses the domain of newspaper articles, magazine articles, and business letters. They sum up the factors in four major classes, which are: 
Imaging Defects are defects introduced along the way between the printing process and the page image submitted to OCR.

Similar Symbols: All OCR devices recognise characters primarily by their shape. But shape is an elusive concept, which can lead to OCR confusion.

Punctuation: Capitalisation and punctuation are guideposts in written material much like inflection and phrasing in speech.

Typography considers the problems imposed by the intended appearance of printed text, as opposed to defects that arise in printing, copying or scanning.

Let us make some remarks on our context. For indexing technical drawings, we need to read the content of two fields: a title and an identification number. These fields contain most of the time less than 30 different characters. The OCR engines can not use any context, and the abbreviation used in the fields does not allow the use of a dictionary. Thus Nagy's classification [5], while adapted to newspapers and other regular papers, does not cover all possible sources of errors for the technical drawing indexing. On the other hand, after having observed many samples of images and results, we concluded that the punctuation and similar symbols classes do not have an important impact for our domain. Thus, we have designed our own classification.

\subsection{Our error cause classification}

Following the preceding remarks, we propose our own error cause classification, which starts from the work of [5] and includes two new classes (see Table 1):

Writer is specific to our domain. Writings on technical drawings are often capital hand-written or stencil ones.

Form Processing: as the form processing extracts fields, it may create some specific kinds of imaging problems. [4] describes several issues in OCR processing, and proposes several solutions. Part of this class draws from [4].

\begin{tabular}{|l|l|l|l}
\hline \multicolumn{1}{|c|}{ Imaging Defects } & \multicolumn{1}{c|}{ Typography } & \multicolumn{1}{c|}{ Writer } & Form Processing \\
\hline 1. Heavy Print & 5. Italic & $\begin{array}{l}\text { 11. Regular Skew of } \\
\text { Chars between } \\
\text { Chars }\end{array}$ & $\begin{array}{l}\text { 18. Character } \\
\text { Touching }\end{array}$ \\
\hline 2. Light Print & 6. Spacing & 12. Regular Spacing & $\begin{array}{l}\text { 19. Damaged } \\
\text { Characters }\end{array}$ \\
\hline 3. Stray Marks & 7. Underlying & $\begin{array}{l}\text { 13. Constant } \\
\text { Typography for } \\
\text { each Font }\end{array}$ & 20. Line Touching \\
\hline 4. Speckle & 8. Very Large Print & 14. Font is Stencil & 21. Vertical Lines \\
\hline & 9. Very Small Print & $\begin{array}{l}\text { 15. Font is Human } \\
\text { Curves }\end{array}$ & 22. Horizontal Lines \\
\hline & $\begin{array}{l}\text { 10. Base Line } \\
\text { Respect }\end{array}$ & $\begin{array}{l}\text { 16. Great Difference } \\
\text { of Font Size }\end{array}$ & 23. Holes, Symbols \\
\hline & & $\begin{array}{l}\text { 17. At least two } \\
\text { Information Classes }\end{array}$ & 24. Image Truncated \\
\hline & & \multicolumn{2}{|l}{}
\end{tabular}

Table 1: classification of error causes

\section{$4 \quad$ Experiment and Analysis}

We build up a database of 250 fields from technical drawings. For each drawing, two kinds of fields are needed for the indexing: a title (alphanumeric) and an ID number (entirely numeric). The extraction of those two fields is automatically done using a methodology that we have developed [6]. We add to the database a $25^{\text {th }}$ parameter specifying if the field is an ID number. For each field, we associate its image parameters and a correction cost with respect to a given OCR engine. We test two Caere OCR engines, a hand-written one and a type-font one. We choose to analyse our database through Principal Component Analysis (PCA) where the correction cost was quantified to 4 levels, from nothing to high. Figure 1 shows the cross cost table for the two OCR engines. On the horizontal is the type-font expert, and on the vertical is the hand-written expert. About $60 \%$ of the images are located in the cluster 1 , which means that for most of the images, some work is needed to correct the OCR results.

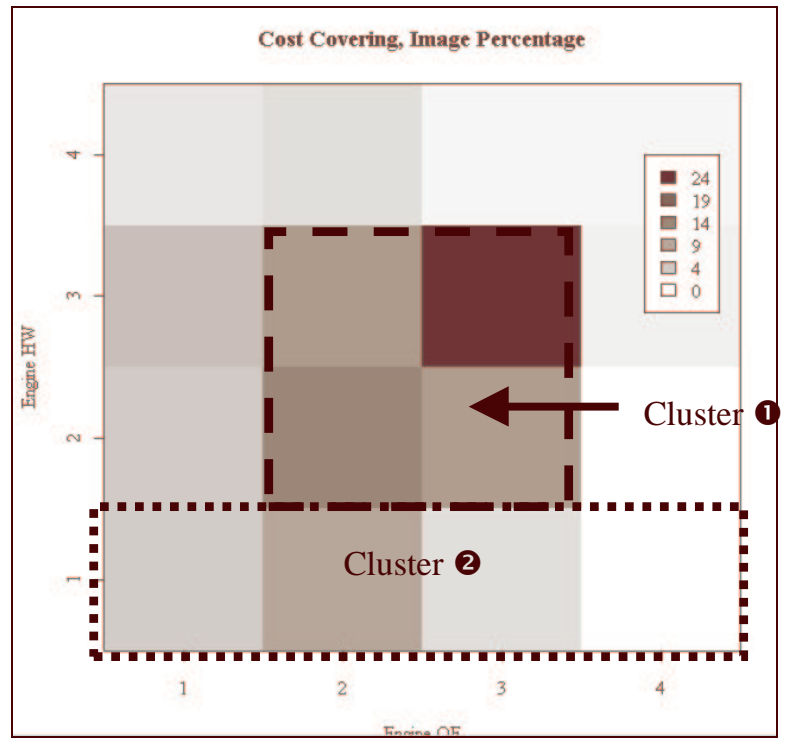

Figure 1: Cross cost table for two OCR engines

We are going to show the analysis of the two clusters represented on figure 1 . 


\subsection{Interpretation of Cluster 1}


Figure 2: Principal Components of $60 \%$ of images

The Figure 2 is divided into four parts. The upper parts show the importance of the first principal components (PC). On the left is the variance of each PC and the right figure gives the cumulative percentage along PCs. The PCs are given for the cost cluster selected by the benchmark operator. This cluster has got an importance because it reflects an OCR "trend" concerning what is studied (result, particular cases, ...). The lower images represent the four first clusters, plotted two by two (PC1 with PC2, and PC3 with PC4). On the scales of those figures are represented the variance for each of the 25 parameters. Those graphs will help the operator to discover among the PCs that represent the majority of cases, which are the predominant parameters. The most important results we can obtain from the PC1, are related to the writer: parameter 15 (Font is Human Curves) is correlated with Regular Skew of Chars (11), Regular Spacing between Chars (12), Constant Typography for each Font (13), Font is Stencil (14)). This means that our OCR engines do not deal perfectly with the specific writing of technical drawings. To correct this problem, a learning phase could be of interest, but such a mechanism is not available for our OCR engines. This is a strong argument to change our OCR engines.

From the PC2, we learn that recognising the title (25) is difficult when the characters touches each other (18), even in the absence of Stray Marks (3).

The third PC teaches us that lines touching characters (20) generate errors. A line removal algorithm could reduce this influence.
From the PC4, we see other form processing problems. In that case, the vertical lines combined with stray marks are the reason. Some algorithms exist that could suppress the problems.

Now that we have seen some important reasons for the OCR recognition failures, we are going to analyse in which conditions the expert devoted to the recognition of human writing works well. This leads us to study the cluster 2 .

\subsection{Analysis of cluster 2}

Let us look at the conditions required for the handwritten expert to work correctly. PCA of cluster 2 of figure 1 is represented in figure 3.


\section{Figure 3: PCA of cluster 2}

On the PC1, we first see that the OCR engine works correctly if the font is human curves $(11,12,13$, and 14 with negative correlation). This is to be expected for an engine devoted to the recognition of handwriting.

From the PC2, we learn that the engine works correctly when reading numbers (25), without speckle (4), with separated characters (18), not in italic (5). The presence of Stray Mark (3) does not seem to be a problem.

The interpretation of the other PCs is hard. Too few images are present on these PCs, and thus it does not allow us to conclude.

As a conclusion, we see that the hand-written engine is an expert that works well in the case of numbers relatively clean. It does not perform as well on alphanumeric text. 


\section{Conclusion}

We have developed a methodology allowing the analysis of strengths and weaknesses of commercial OCR engines seen as black boxes. Lead by the integration in the global digitisation chain, we model the behaviour of an OCR engine as a function of image characteristics returning a correction cost:

For each image we associate some parameters describing what could lead to OCR errors. Those parameters are adapted to the technical drawing domain;

A human cost of correction is attributed to each OCR result.

We analyse the behaviour of the OCR engines through Principal Component Analysis. While we believe this methodology to be generic, we applied it to the Technical Drawing domain, with two OCR experts from CAERE Corporation. One OCR is devoted to type-font and the other one to hand-font. We note that around $60 \%$ of images are not correctly recognised. For those images, we found that the most useful operation would be to learn the specific technical drawing font, an operation that is not feasible with our OCR engines. The second most annoying problem is touching characters. Then came some form processing issues. Stray Marks do not seem to be an important problem. On the other hand, analysing the behaviour of the hand-font expert, we conclude that this OCR engine performs better on numerals than on alphanumeric text. That confirms the importance of the learning phase. Thus, while at the beginning of our study we thought that there was no need to develop our own OCR, we find that we should at least choose an OCR engine with learning capabilities.

Now that we are able to determine OCR skills, we could develop an OCR combination methodology based on the knowledge of those skills. In the current literature, this knowledge is limited to correlation among experts. Our benchmarking technique provides much more information. One way to exploit this information is to design parameter estimators that will allow choosing the OCR adapted to a given image.

The authors would like to thanks A. Belaid for numerous helpful discussions while working on this subject.

\section{Bibliography}

[1] Abdel Belaid, L. Pierron, L. Najman, D. Leyren "La numérisation de document: principes et evaluation des performances." chap. 2 in Bibliothèques numériques, pp. 53-98. ADBS éditions, 2000

[2] J. L. Blue, G. T. Candela, P. J. Grother, R. Chellappa, C. L. Wilson, J. D. Blue. "Evaluation of Pattern Classifiers for Fingerprint and OCR Application", in Pattern Recognition, 27, pp. 485501, 1994

[3] Dengel, R. Hoch, F. Hones, T. Jager, M. Malburh, A. Weigel "Techniques for improving OCR results", Handbook of Character Recognition and Document Image Analysis, pp 227-258, 1997 World Scientist Publishing Company.

[4] R.R. Hashemi, D. Owen, M.S. Smith, T. Flanigan and J.R. Talburt. "The effect of Image Enhancement in OCR systems: A Prototype". Proceedings of the International Conference on Information Technology: Coding and Computing, 2000 (ITCC'00)

[5] G. Nagy, T. Nartker, S. Rice "Optical Character Recognition: An illustrated guide of the frontier", SPIE Vol. 3967, January 2000

[6] L. Najman, O. Gibot and S. Berche "Indexing Technical Drawings using Title Block Structure Recognition". ICDAR 2001, to appear in this issue

[7] T. Narkter "Benchmarking DIA Systems " Handbook of Character Recognition and Document Image Analysis”, pp 801-820, 1997

[8] C. L. Wilson, J. Geist, M. D. Garris, R. Chellappa, "Design, Integration, and Evaluation of FormBased Handprint and OCR Systems", Technical Report NISTIR 5932, December 1996.

[9] C. L. Wilson. "Evaluation of Character Recognition Systems," In Neural Networks for Signal Processing III, IEEE, New York, pp. 4854961993. 